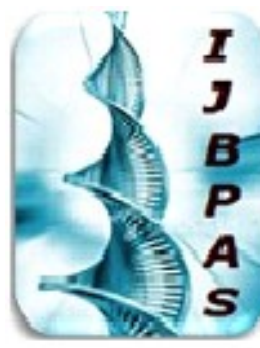

International Journal of Biology, Pharmacy and Allied Seiences (IJBPAS)

'A Bridge Betueen Caboratory and QRendo'

WwW.ibpas.com

\title{
ACCURACY AND PRECISION OF BLOOD GLUCOSE METERS IN \\ PONDICHERRY AND TIRUPATI IN SOUTH INDIAN POPULATION
}

\section{E. PRABHAKAR REDDY ${ }^{*}$, A.VAITHIALINGAM ${ }^{2}$, T.MOHANA LAKSHMI ${ }^{3}$, R. E. PURUSHOTHAM REDDY ${ }^{4}$}

1: Professor in Biochemistry, Bharath Medical College and Hospital, Chennai and Affiliated to BIHER

2: Department of Orthopedics, Sri Lakshmi Narayana Institute of Medical Sciences, Puducherry, BIHER, India

3: Department of Microbiology, Sri Lakshmi Narayana Institute of Medical Sciences, Puducherry, BIHER, India

4: Arogya Diagnostic Centre, Tirupati, Andrapradesh, India

*Corresponding Author: Dr. E. Prabhakar Reddy: E Mail: drpebyreddy@yahoo.com Received $17^{\text {th }}$ Oct. 2020; Revised $16^{\text {th }}$ Nov. 2020; Accepted $12^{\text {th }}$ Dec. 2020; Available online $1^{\text {st }}$ Jan. 2021

\section{https://doi.org/10.31032/IJBPAS/2021/10.1.1013}

\section{ABSTRACT}

Since the introduction of glucometers, there has been an ongoing, competition-driven development in both meter and strip technology, which glucometer is greater accuracy and reliability of results. Despite the advances in technology, there is significant variation amongst these glucometers necessitating a proper evaluation before use. Glucose levels in glucometers in blood samples 200 patients attending in Arogya diagnostics at Tirupati and SLIMS Hospital at Puducherry were measured with each meter, and compared with the laboratory reference method. To assess the accuracy and precision of 2 currently available blood glucose meters in Pondicherry and Tirupati. The coefficients of variation (CVs) of most meters were acceptable at less than $6 \%$. With the introduction of glucometers, there has been an ongoing, competition-driven development in both meter and strip technology, which has allowed for greater accuracy and reliability of results. Although most emergency units and tertiary hospitals have access to laboratory glucose measurements. It is therefore 
imperative that glucometer values are accurate and precise at important medical-decision thresholds. A failure in this regard may lead to critical medical errors. The study highlights the need for an objective and independent comparison of all glucometers in South India, as the variability observed can impact on patient care.

\section{Keywords: Glucometers, Blood Glucose, Accuracy and reliability, Centralized}

\section{laboratory}

\section{INTRODUCTION}

Glucose meters are now widely used in hospitals for monitoring blood glucose at the bedside. Changes in medical practice have intensified institutional pressures to achieve clinical efficacy. Thus hospitals are decreasing the admission of patients with non-acute conditions and increasing the proportion of patients admitted for major therapeutic interventions [1]. With the introduction of glucometers, there has been an ongoing, competition-driven development in both meter and strip technology, which has allowed for greater accuracy and reliability of results [2]. However, despite the advances in technology, there is significant variation among these monitoring devices, which has necessitated the development of performance guidelines by organizations such as the International Standardization Organization (ISO) [3]. These glucometers have traditionally been subjected to less rigorous analytical requirements. Even though the precision and accuracy of most meters have improved over the years, there are still concerns regarding the standardisation of these glucometers and their failure in satisfying the recommendation of less than $5 \%$ deviation when compared to a reference method. It also allows medical personnel and patients to choose glucometers more objectively, thereby improving the quality of care.

Continuous glucose monitoring provides information about the direction, magnitude, duration, frequency, and causes of fluctuations in blood glucose levels. Compared with conventional intensified glucose monitoring, defined as three to four blood glucose measurements per day, continuous monitoring provides much greater insight into glucose levels throughout the day. Continuous glucose readings that supply trend information can help identify and prevent unwanted periods of hypo- and hyperglycemia. Situations that require detailed information about blood glucose fluctuations that only continuous monitoring can provide include when adjusting therapy, quantifying the response in a trial of a diabetes therapy, assessing the impact of lifestyle modifications on 
glycemic control, monitoring conditions where tighter control without hypoglycemia is sought (e.g. Gestational diabetes, pediatric diabetes, in the intensive care unit), diagnosing and then preventing hypoglycemia (e.g., during sleep, with hypoglycemia unawareness), and diagnosing and preventing postprandial hypoglycemia. The most important use of continuous blood glucose monitoring is to facilitate adjustments in therapy to improve control. Continuous glucose monitoring offers the capability of expressing the frequency and severity of hypoglycemic episodes much more clearly than does intermittent glucose testing. Continuous glucose monitoring also offers the capability of expressing the mean blood glucose value in new ways. Continuous glucose monitoring can document the time spent in the normal, low, and high ranges, which may be more valuable than a single integrated data point. Methods for minimally invasive and noninvasive continuous monitoring advance, diabetic patients will use this technology more routinely.

Diabetes mellitus is a disease reaching epidemic proportions globally. It is predicted that the impact on the developing world will soon outweigh that on the developed world. In the year 2000, the developing world accounted for $72.5 \%$ of the world total of diabetes sufferers. Bedside blood glucose testing using reagent impregnated strips and simple reflectance meters has been enthusiastically accepted as quick and simple means to monitor blood glucose levels [4]. The importance of the self-monitoring of blood glucose using home blood glucose meters has prompted numerous reports in scientific literature regarding the statistical and clinical accuracy of these devices [5]. The availability of sophisticated dry and wet chemistry systems that offer a sizeable menu of laboratory tests has made it possible for laboratory tests to be done outside the central clinical laboratory [6]. Accuracy is the ability of a test to produce results close to the best available measure. Turnaround time (TAT) is a complex process that begins with the phycisician's initiation of a laboratory order, continues with the acquisition of the appropriate specimen, proceeds with the actual analysis time, and concludes with the transmission of the results to the physician [7]. To determine accuracy, turnaround time and cost effectiveness, we performed blood glucose levels in the central laboratory on automated analyzer and at bedside by nonlaboratory health care professionals with glucometer.

Glucometer may not be very accurate across the full range of glucose 
values, especially lower values, its utility however, as a screening tool cannot be underestimated [8]. Keeping in mind their widespread utility we conducted the study to compare the analytical performance, turnaround times and cost effectiveness of the two glucose testing modalities. This number is projected to double in subSaharan Africa by the year 2030 [9]. Very few epidemiological studies regarding the prevalence of diabetes have been conducted and reported in South Africa since 1994. One of the landmark studies in 1998 estimated the prevalence of self-reported diabetes in the age group older than 15 years to be $2.4 \%$ and $3.7 \%$ among males and females respectively. This study also revealed racial and geographic variations in the prevalence of diabetes. In another study, Levitt and co-workers demonstrated an age-standardised prevalence of $10.8 \%$ of type 2 diabetes in the age group 30 to 65 years in a coloured community in Mamre, Cape Town. With the rising rates of obesity and metabolic syndrome in the young and with the urbanisation of African communities, it is clear that the number of people with diabetes will increase. Finding in our study suggest that very low and high glucose values with glucometer do not accurately reflect actual plasma glucose levels. In order to make critical decisions at these levels, plasma glucose should be confirmed with centralized laboratory based methods. The bedside glucose testing with glucometer is a simple, rapid, cost effective method for glucose monitoring.

The test strips are one-time use only, are disposed of after each individual measurement, and cost about 17 Rs each depending on the model of the glucometer. Glucometers cost approximately 1000 to 2000.For instance, do different glucometers give the same value statistically for the same standard sample. Third, if inconsistencies such as two glucometers giving different results for the same sample are found, experiments can be designed with the oversight of the instructor to determine the cause of inconsistencies. To help illustrate these points' examples from data obtained performing the same analysis on the same aqueous glucose sample using two different glucometers. Taken individually, each set of data is very representative of the precision obtained by labs using the glucometers. Examining the mean values obtained by each glucometer appears that labs, using glucometer 1 and 2, has measured glucose concentration values that appear to be statistically significant of $15 \%$. Performing a $t$-test allows this to be proven. After determining that these data sets are indeed different, the obvious question in lab data which glucometer is which set of data is more accurate until this 
point in the discussion, which takes place after the experiment has concluded, the concentration of the glucose standards is not revealed. In general this is done to prevent the data from being biased and to give the instructor flexibility in the learning process. For the case presented here, the standard sample being measured was a pure aqueous solution of glucose with a concentration of $200 \mathrm{mg} / \mathrm{dL}$. Therefore, it is evident that both readings that are low compared to the "actual" value.

To determine if there are statistical differences from the "actual" concentration, one-population $t$-tests can again be performed in comparison to the nominal value. For both sets of data there is a statistical $15 \%$ difference.

\section{MATERIAL AND METHODS}

Glucose levels in glucometers in blood samples 200 patients attending in Arogya diagnostics at Tirupati and SLIMS Hospital at Puducherry were measured with each meter, and compared with the laboratory reference method.

\section{Aim of the Study}

To assess the accuracy and precision of 2 currently available blood glucose meters in at Puducherry and Tirupati.

\section{RESULTS}

Results of same samples using 2 different GM: (Table 1).

Measured glucose concentration $\mathrm{mg} / \mathrm{dl}$.

Table 1: Results on the same sample using 2 different glucometer

\begin{tabular}{|c|c|c|c|c|c|}
\hline \multicolumn{2}{|c|}{} & \multicolumn{2}{c|}{ Glucometer 1 } & \multicolumn{2}{c|}{ Glucometer 2 } \\
\hline S.No. & Age & Male & Female & Male & Female \\
\hline 1 & 43 y & 127 & 123 & 135 & 139 \\
\hline 2 & 38 y & 118 & 130 & 150 & 163 \\
\hline 3 & 50 y & 128 & 142 & 139 & 120 \\
\hline 4 & 53 y & 120 & 138 & 143 & 118 \\
\hline 5 & 54 y & 125 & 130 & 136 & 152 \\
\hline 6 & $49 y$ & 123 & 118 & 132 & 128 \\
\hline Mean \pm SD & & $124.4 \pm 3.3$ & $140 \pm 6.5$ & $\mathbf{1 3 8 . 5} \pm 6.4$ & $146 \pm 7.9$ \\
\hline
\end{tabular}

\section{DISCUSSION AND CONCLUSION}

Continuous glucose monitoring offers advantages over intermittent glucose monitoring when glycemic patterns are poorly understood. The information about direction, magnitude, duration, frequency, and causes of fluctuations in blood glucose levels that can be obtained by continuous glucose monitoring is simply not available with intermittent blood glucose monitoring. Often results can lead to in-depth questions and further experiments which focus on error analysis and the reliability of the glucometers. The measurement is both stimulating and relevant to the students. The major drawback in this experiment is the expense of the meters and test strips. Previous studies the Diabetes Research in 
Children Network (DirecNet) is a U.S. network of five clinical centers and a coordinating center dedicated to researching glucose monitoring technology in children with type 1 diabetes [10]. Now a days in India also it is increased. The burden and economical strain of this disease due to associated complications, such as cardiovascular and renal disease, is estimated to increase dramatically and could consume as much as $40 \%$ of some countries' health budgets [11]. This increase will probably lead to an increased usage of glucometers in the home care, clinic and emergency care setting. It is also estimated that more models of glucometers will be introduced into the market in response to this demand. With the introduction of glucometers, there has been an ongoing, competition-driven development in both meter and strip technology, which has allowed for greater accuracy and reliability of results [12].

There is significant variation among these monitoring devices, which has necessitated the development of performance guidelines by organisations such as the International Standardization Organization (ISO) [11]. The ISO guidelines recommend that the total analytical error of the glucometers be within $\pm 0.83 \mathrm{mmol} / \mathrm{l}$ of the laboratory blood glucose concentrations when values are $<4.2 \mathrm{mmol} / 1$. The variability observed with glucometers can impact on patient care in different settings, some of which include the diabetic patient on insulin in a home care or clinic setting, and emergency care units in tertiary hospitals. Frequent glucose determinations and insulin adjustments are made according to glucometer readings. Inaccuracies can lead to misclassification of hypo- or hyperglycemic episodes. Although most emergency units and tertiary hospitals have access to laboratory glucose measurements. It is therefore imperative that glucometer values are accurate and precise at important medical-decision thresholds. A failure in this regard may lead to critical medical errors. The Clarke error grid analysis revealed that all glucometers demonstrated adequate clinical accuracy, with most measurements. Accu-Chek ${ }^{\circledR}$ Active and other company One touch horizon recorded one and two measurements respectively. It is not known whether this finding is significant, as these two meters are calibrated to whole blood rather than plasma. As all glucometers were shown to have a proportional bias. Although we were unable to include measurements in the hypoglycemic range, this study highlights the need for an objective and independent comparison of all glucometers available in India. It also allows medical personnel and 
patients to choose glucometers more objectively, thereby improving the quality of care.

The study highlights the need for an objective and independent comparison of all glucometers in South India, as the variability observed can impact on patient care.

\section{Conflict of Interest- Nil.}

REFERENCES

[1] Jacobs E, Vadasdi E, Sarkozi L, Colman N. Analytical evaluation of iSTAT Portable Clinical Analyzer and use by nonlaboratory health-care professionals.Clin Chem. 1993; 39(6): 1069-74.

[2] Cohen M, Boyle E, Delaney C, Shaw Jonathan. A comparison of blood glucose meters in Australia. Diabetes Research and Clinical Practice 2006; 71: 113-8.

[3] Rheeder P. Type 2 diabetes: The emerging epidemic. SA Fam Pract. 2006; 48(10) page 20.

[4] Greendyke RM. Cost analysis. Bedside glucose testing. Am J Clin Pathol 1992; 97(1): 106-7.

[5] Trajanoski Z, Brunner GA, Gfrerer RJ, Wach P, Pieber TR. Accuracy of home blood glucose meters during hypoglycemia. Diabetes Care 1996; 19(12): 1412-15.

[6] Nanji AA, Poon R, Hinberg I. Nearpatient testing. Quality of laboratory test results obtained by non-technical personnel in a decentralized setting. Am J Clin Pathol 1998; 89(6): 797-01.

[7] Nosanchuk JS, Keefner R. Cost analysis of point-of-care laboratory testing in a community hospital. Am J Clin Pathol 1995; 103(2): 240-43.

[8] Hamid MH, Chishti AL, Maqbool S. Clinical utility and accuracy of a blood glucose meter for detection of neonatal hypoglycemia. J Coll Physicians Surg Pak 2004; 14(4): 225-28

[9] Wild S, Roglic G, Green A, Sicree R, King H. Global prevalence of diabetes: Estimates for the year 2000 and projections for 2030. Diabetes Care 2004; 7: 1047-53.

[10] Weinzimer SA, Tamborlane WV, Chase HP, Garg SK: Continuous glucose monitoring in type 1 diabetes. Curr Diab Rep 2004; 4: 95-100.

[11] Rheeder P. Type 2 diabetes: The emerging epidemic. SA Fam Pract. 2006; 48(10) page 20.

[12] Cohen M, Boyle E, Delaney C, Shaw Jonathan. A comparison of blood glucose meters in Australia. Diabetes Research and Clinical Practice 2006; 71: 113-8. 